\title{
COGNICIÓN Y TRANSACCIÓN ESTÉTICA: UN ENFOQUE PARA LA ENSEÑANZA DE LA LECTURA
}

\author{
Marta E. Sánchez Salazar
}

Resumen: Se discute aquí sobre la importancia de enfatizar el uso de metodologías de orden estético transaccional, como complemento de los métodos de enseñanza de la lengua materna los que, en la práctica, se han concentrado mayormente en el aspecto cognitivo-mecanicista de la lengua. Se argumenta que en la carencia de metodologías transaccionales podría yacer una de las causas de los malos hábitos de lectura en la población. Finalmente, se sugiere un respaldo filosófico y metodológico bidireccional, por medio de la teoría de la transacción estética de Rosenblatt y la teoría del esquema.

Palabras clave: comprensión lectora, literatura, transacción, interacción, metodologías.
Nadie puede enseñarte nada que no se halle ya medio dormido en los albores de tu conocimiento.

(K. Gibran)

La presente discusión tiene como objeto señalar la importancia de respaldar los métodos de enseñanza de la lectura con un enfoque bidireccional cognitivo-estético. Se argumenta aquí que la comprensión lectora juega un papel central en las primeras etapas del desarrollo integral infantil, por lo que cualquier aproximamiento didáctico hacia la lectura en etapas tempranas debe considerar, en primer lugar, el "orden natural" que supone la totalidad del proceso de adquisición del lenguaje y en segundo lugar, el componente lúdico, condición sine qua non, del proceso de aprender en el niño. Se establece que parte de la causa de la deserción escolar puede yacer en un enfoque equivocado de la lengua materna en la escuela, ya que, a menudo, se tiende a ver el lenguaje como cualquier otra cosa y no como lenguaje y esto afecta, desde luego, la comprensión lectora. Lo anterior, si se tiene en cuenta que la lectura, como cualquier otra destreza de la lengua, se desarrolla cognitiva, cultural y estéticamente. Se concluye con 
la sugerencia sobre métodos de enseñanza respaldados por la teoría del esquema y la de la transacción estética de L.M. Rosenblatt, considerando que ambos aproximamientos se complementan pese a proceder de paradigmas casi opuestos. Se argumenta que el respaldo teórico que ambos ofrecen, permiten metodologías más adecuadas al complejo constructo que presupone la interacción cognitiva y la transacción estética de aprender a leer.

\section{Antecedentes}

La investigación en el campo del aprendizaje en general, durante la segunda mitad del siglo pasado, trajo consigo cambios radicales en torno al fenómeno de la adquisición y desarrollo del lenguaje. La teorías cognitivas, principales responsables de este giro, pusieron en la picota las teorías conductistas tradicionales. Estas últimas, basadas en la concepción skinneriana de estímulo-respuesta, apoyaban la creencia de que el aprendizaje de la lengua era resultado de una "disposición mimética" estimulada mediante un refuerzo adecuado y repetitivo del cual emergería la respuesta como reflejo condicionado. Más tarde, los principios racionalistas vinieron a poner en tela de juicio el paradigma conductista. N. Chomsky, posiblemente la figura más destacada en esta revolución, propuso la idea de que el aprendizaje de la lengua es innato, resultado de un "dispositivo" natural o LAD -language acquisition device. Éste, como cualquier otro órgano, crecería y se desarrollaría conforme a las condiciones lingüísticas circundantes. A diferencia de los conductistas, los racionalistas verían en el medio, no el condicionador para el desarrollo del lenguaje, sino el generador de un potencial cognitivo existente desde antes del nacimiento del individuo. El mismo Chomsky diría más tarde:
“. . . desde el nacimiento hasta la muerte cada organismo pasa por una serie de cambios programados genéticamente. El desarrollo del lenguaje es simplemente uno de estos cambios predeterminados.

El lenguaje depende de una donación genética paralela a aquellas que especifican la estructura de nuestros sistemas óptico o circulatorio, o que determinan que tengamos brazos en vez de alas" (en RichardAmato, 1988, capítulo 1).

Numerosas metodologías para la enseñanza de lenguas extranjeras, por ejemplo, emergieron a la luz de los principios de Chomsky. Igualmente, investigadores de diferentes áreas del aprendizaje humano comenzaron a cambiar la dirección de sus estudios hacia el enfoque cognitivo-racionalista, viendo en Chomsky casi "un liberador del dogma paralizante contenido en el paradigma asociaciónimitación del conductismo", como lo pondría T. Bruner (en Richard-Amato, p.14). Este contexto teórico-filosófico afectó de manera inmediata toda la investigación acerca del fenómeno de comprensión lectora y los resultados de estos cambios se extienden hasta nuestros días.

La primera y más importante consecuencia del paradigma cognitivo-racional dentro del contexto de la comprensión lectora es que ha dejado al descubierto la necesidad de una mejor adecuación de la enseñanza de la lectura que considere lo afectivo-estético. Es posible que el factor que más haya incidido en una aparente apatía a realizar cambios metodológicos en este campo, descanse en el poco conocimiento teórico existente entre los educadores acerca de la naturaleza del fenómeno de comprensión lectora, tanto desde el punto de vista cognitivo como de transacción estética. En este sentido, hay que decir que, aunque se han realizado numerosos estudios de índole cognitivo-esquemática acerca del acto de lectura, todavía se sabe relativamente poco sobre su naturaleza estético-transaccional, es decir, sobre lo que ocurre "afuera", a nivel de respuesta del lector. Es por esto prudente ofrecer 
aquí un resumen de lo que se entiende por lo cognitivo-racional en oposición a lo transaccional-estético en cuanto al fenómeno de comprensión lectora.

De acuerdo con la teoría de los esquemas el proceso lector se desarrolla como otra modalidad del lenguaje y, como tal, sigue un orden natural. Así, la comprensión lectora es el resultado de una interacción de esquemas de conocimiento entre lector y texto. La construcción del significado activada por la descodificación de los signos lingüísticos tiene lugar, según Rumelhart (1977), conforme la información busca "acomodo" en los esquemata existentes. La falta de los esquemata adecuados puede resultar en la imposibilidad de que la información se almacene en la memoria.

Desde el punto de vista transaccional, el paradigma de la teoría de la respuesta del lector (Reader Response Theory) es el más conocido. Una de las pioneras de este enfoque, Louise Rosenblatt, propone la Teoría de la Transacción Estética que enfoca no lo cognitivo, sino más bien lo "visible", es decir, la respuesta resultante de una transacción de visiones de mundo entre autor y lector. Rosenblatt considera el componente estético inherente al proceso de lectura. La interpretación deviene en respuesta, el producto que trasciende lo meramente cognitivo-mecanicista, para elevarse al plano de lo estético-vital, en una palabra a la experiencia. En este intercambio, la unión del lector y el texto crean un tercer elemento que, en el caso de la interacción con el texto literario, según palabras de Rosenblatt (1978), puede llamarse con el nombre genérico de "Poema."

Desde la perspectiva de esos principios de conocimiento y de experiencia de vida, el fundamento filosófico sobre el que debe descansar la metodología de la enseñanza de la lectura se ha de definir como lo que es ese fenómeno: un proceso en constante desarrollo que encuentra un campo fértil en la disposición innata que el cerebro humano parece tener respecto del lenguaje en sus diferentes modalidades. Ignorar esta perspectiva significaría romper o alterar la cadena natural del aprendizaje. Al igual que el concepto de innateness propuesto por Chomsky en referencia a la disposición natural hacia el habla, la materia prima de la comprensión lectora yace en las estructuras cognitivas que permiten que, del conocimiento previo del lector y su visión de mundo, se deriven su capacidad para predecir, hipotetizar y jugar con la interpretación, todo como producto del "poema".

El proceso de comprensión de lectura comienza aún antes de la articulación de las palabras, de aquí que podría especularse que la comprensión lectora es un proceso que se da "al margen" de la instrucción formal. Nuevamente, de acuerdo con Chomsky, tenemos que el niño nace con un potencial genético hacia el lenguaje que incluye el componente semántico. J. Lindfors (1980) describe este proceso en el infante como sigue:

"Las cosas de su ambiente son lo que él sea capaz de hacer con ellas ... es a través de la acción física del niño sobre su ambiente que su visión de la realidad cambia: él se mueve desde una percepción de la realidad como un continuo de visiones, aromas, sonidos, gustos y sensaciones táctiles que incurren en sus órganos sensoriales desde su nacimiento, hacia una percepción de la realidad como entidades, acciones y personas separadas espacialmente" (p. 166, mi traducción).

En el mismo sentido y en relación con la aprehensión del lenguaje impreso por parte del infante, M. Jager (1990) agrega: "En algún momento, los niños deben intuir que la escritura es categóricamente diferente de cualquier otro patrón visual presente en su entorno" (p. 334, mi traducción). Jager sugiere que la atención del niño hacia lo impreso circundante podría ser bien el primer paso crítico hacia la lectura, el paso que provee al niño tanto la comprensión como el incentivo necesarios para acercarse al material impreso descontextualizado de los libros de texto (p. 341). 
Comprender la lectura como una modalidad más de la adquisición del lenguaje impone la necesidad de conocer más de cerca el desarrollo de este proceso en el niño y la proyección gradual que éste efectúa de su mundo interno hacia el material impreso. Como la adquisición del lenguaje es producto de la interacción constante del cerebro con el entorno, el proceso de adquisición de la lengua está en constante desarrollo, ya que el mundo circundante es una inagotable fuente de información para el niño. Como respuesta inmediata, el infante comienza a crear su propio mundo "paralelo" formado, organizado y modificado una y otra vez, con base en nuevas e incesantes experiencias de vida. Todo este proceso es el resultado de un "misterioso" conocimiento previo (schemata) producto de un continumm cognitivo por el que todo aprendizaje es posible, según los teóricos del esquema. En esta misma línea, afirma F. Smith (1988): "Los niños podrían no siempre encontrar fácil o aún necesario aprender lo que nos esforzamos en enseñarles, pero encuentran la condición de no aprender del todo, intolerable" (mi traducción).

Son numerosos los estudios cognitivos que demuestran que los niños, independientemente de su IQ, son capaces de desarrollar destrezas lingüísticas de forma creativa y con una velocidad sorprendente, así como de manejar estructuras complejas desde muy tempranas etapas (Adams, M., 1990; Applebee, A., 1989; Chomsky, N., 1988; Cochran-Smith, 1986; Downing, J., 1979; Pearson, B., 1996; Smith, F.,1988). Lo anterior, una vez más, implica la existencia de un sistema innato de principios subyacentes cognitivamente que permiten al niño captar hasta los significados "ocultos" de palabras y expresiones. Lindfors describe situaciones en las cuales el niño se muestra sensible al contenido semántico de la lengua. Por ejemplo, a un grupo de niños se les dio una lista de palabras desconocidas cuyo significado podían deducir sólo si se les enseñaba dentro de un contexto comprensible. Pero si las palabras se ofrecían aisladas, los niños ni siquiera podían pronunciarlas bien (p. 222).

El hallazgo de Lindfors es digno de considerar a la hora de evaluar las prácticas metodológicas convencionales. No es secreto que, a menudo, muchas de ellas someten a los niños de primero y segundo grado a respectivos ejercicios descontextualizados de "lectura" en los que se privilegia o la división silábica por un lado, o la lectura para extraer un detalle informativo por el otro. Ambas prácticas, aunque complementariamente necesarias, distan considerablemente de la verdadera naturaleza estética del acto lector. (Ver apéndice). Es posible que esta descontextualización tenga efectos negativos en el desarrollo del aprendizaje en general, sobre todo si el proceso enseñanza-aprendizaje se hace depender desproporcionadamente de ese enfoque. Por ejemplo, una historia de la cual el niño siempre aprende algo, se sacrifica, se mutila, cuando es usada solamente en aras de la parte mecánica de la lengua materna. De este modo, el orden natural de adquisición del lenguaje, como experiencia vital, se ve interrumpido desde etapas tempranas del aprendizaje formal.

En relación con el tipo de instrucción tradicional o programada alrededor de lo meramente cognitivo, las observaciones de Lindfors tienen otras claras implicaciones. Para comenzar, hay que recordar que el proceso de comprensión yace en la raíz del conocimiento humano y, por lo tanto, la instrucción programada difícilmente podrá ajustarse a esa compleja realidad. Si se parte del hecho de que los niños de seis y siete años están lo suficientemente maduros como para recibir instrucción lectora pero no lo están todavía para enfrentar la lengua materna de forma "objetiva", es decir, como un elemento ajeno a su naturaleza, tenemos que las técnicas de enseñanza de lectura deberían, por necesidad, girar estrictamente en torno a ese proceso 
natural y no separado del mismo. En otras palabras, los programas de aprendizaje y desarrollo de la comprensión lectora tienen, por necesidad, que partir de esos principios epistemológicos primordiales.

Leer un lenguaje es también lenguaje y por lo tanto, debe enfocarse siempre como tal, respetando el potencial cognitivo que las diferentes metodologías intentan, en principio, desarrollar en el niño. Sin embargo, más a menudo de lo deseable, la instrucción tradicional reflejada en lo que muchos maestros hacen en el aula parece ignorar esos principios epistemológicos. Y es que el proceso de comprensión lectora frecuentemente se ha enfocado a la inversa. Se le tiende a ver como el resultado de "saber leer" y no como una disposición natural para aprender a leer. Tal parecen sugerir, repetimos, algunos métodos de instrucción tradicionales, basados en la división silábica más que en el contenido de la narrativa coherente. $\mathrm{Y}$, como se argumentó antes, mediante estos métodos se enfrenta al niño con complejos mecanismos que presuponen un aún más complejo proceso de metacognición, en etapas en que el cerebro joven todavía no ha desarrollado las destrezas necesarias para tal efecto. De este modo, se obliga al niño a mirar el lenguaje como un ente "ajeno" a su mundo. Al respecto, Lundberg y Torneus consideran irrelevantes a la verdadera naturaleza del lenguaje, las prácticas metodológicas de lectura consistentes en descomponer la lengua en unidades de sílabas, o vocabulario y frases descontextualizados. En su opinión, dichas prácticas ponen en riesgo el proceso de aprendizaje en su totalidad, pues se implantan bastante antes de que los niños hayan desarrollado las destrezas metalingüísticas necesarias (en Downing, p. 18, 1979). Es obvio que los niños necesitan instrucción programada para desarrollar la lectura y la escritura. No obstante el énfasis en cierto tipo de prácticas didácticas puede inhibir la disposición natural en el cerebro del niño para desarrollar las destrezas cognitivas, en concordancia con la misma disposición que permitió el desarrollo del habla. En realidad, la naturaleza cognitiva va a la par de la naturaleza estética del proceso de comprensión lectora, por lo tanto, el proceso de lectura debe desarrollarse mediante técnicas alternativas que privilegien tanto lo cognitivo como lo estético.

Dado que el lenguaje es una extensión del mundo interno del niño, se entiende que cualquier representación gráfica de la lengua es sentida por éste como otra manifestación de ese mundo. Es así que puede decirse que leer es para el niño una necesidad disfrazada de mezcla de curiosidad intelectual e instinto. Leer le ayuda a organizar su "caos", como lo diría F. Smith. Las palabras, los números, las figuras geométricas, parte de un mundo en el que su mundo interno se encuentra inmerso, son elementos que el niño reconoce cuando los ve por primera vez porque, detrás de este reconocimiento, hay un conocimiento previo provocado por el medio circundante que le capacita para predecir, condición esencial para la comprensión lectora. M. Strange (1980), citando a Adam \& Bruce, dice:

"En realidad, la comprensión lectora involucra la construcción de ideas a partir de conceptos preexistentes. Una afirmación correcta del papel del conocimiento antecedente sería que la comprensión es el uso del conocimiento previo para crear nuevo conocimiento. Sin conocimiento previo, un objeto complejo tal como un texto, no es sólo difícil de interpretar, estrictamente hablando, sino que carece de significado por completo" (395, mi traducción).

La comprensión lectora involucra también un propósito personal sin el cual la lectura per se no tendría sentido. Este propósito está cercanamente relacionado con la capacidad natural del lector de predecir. F. Smith explica que es precisamente esa capacidad la que nos hace funcionales en nuestro mundo. En el contexto de la interacción texto-lector, la condición innata de la predicción de parte del lector podría explicar la habilidad del niño para entender 
el lenguaje metafórico. R. Billow (en Applebee, 1989), uno de los estudiosos en este campo, encontró que antes de los cinco años, el niño puede entender intuitivamente un significativo porcentaje de metáforas sin la ayuda de procesamientos operacionales. Afirma Applebee:

"El estudio de Billow es particularmente interesante (e inusual) en su cuidadoso análisis de la operaciones en las que se involucran las metáforas de varios tipos y en las relaciones entre estas operaciones y la habilidad del niño para resolver problemas similares en los que la metáfora no está involucrada” (p. 104, mi traducción).

Es decir, el cerebro joven es capaz de procesar la metáfora tan bien como su equivalente literal, lo que refuerza el hecho de la comprensión del lenguaje figurativo entre los preescolares. F. Smith (1988) ve en esta predisposición natural la clave del misterio del por qué algunos libros para niños resultan tan exitosos. Es como si la necesidad que tiene el niño de encontrar respuestas a sus preguntas los hiciera adivinar el significado que hay detrás de las palabras mediante el uso de su conocimiento previo y su capacidad para predecir.

"Vivimos en un mundo creado por el cerebro, continúa Smith, y no en un mundo existente al margen nuestro... la lectura puede proporcionar verdaderas experiencias en mundo reales, no meras réplicas de experiencias en 'representaciones' del mundo. El mundo en la cabeza es la base de toda nuestra realidad" (F. Smith, p. 224, mi traducción).

Vista desde la perspectiva que podemos denominar estético-cognitiva, la interacción-transacción lectora va más allá de la palabra impresa. Es, más bien, el resultado de una disposición de parte del lector para recibir y responder a la palabra impresa, razón por la que los primeros años de instrucción lectora son de importancia fundamental para todo el proceso de aprendizaje.

Se afirma que el lector siempre tiene un propósito ante un texto dado, el cual logra únicamente si el texto le dice algo a él.
Esto sólo puede ocurrir si el discurso aparece estructuralmente en concordancia con la disponibilidad cognitivo-esquemática del lector. Es decir, un lector joven necesita una preparación esquemática que le permita procesar un texto de naturaleza expositiva, por ejemplo. De aquí que las primeras experiencias lectoras son fundamentales para el éxito del procesamiento de otros textos, como los expositivos o argumentativos con que el escolar se enfrentará más tarde.

Como se sabe, la naturaleza de las experiencias primordiales es episódica y va formando los esquemas de conocimiento en un orden equivalente. Es por esto que los primeros textos con que se enfrenta el niño deben contener el esquema narrativo, la estructura más cercana a la disposición cognitivo-esquemática del lector novato. En este principio yace el "misterio" del por qué las historias y cuentos de hadas pueden ser procesados por el cerebro del niño de forma relativamente fácil. En otras palabras, el discurso episódico o narrativo es más fácilmente procesado por el cerebro que cualquier otro tipo (Applebee, 1989). "La simplicidad de las historias que cuentan los niños, explica A. Applebee, ayuda a dar luz a los principios [cognitivos] que subyacen a su forma”, (p. 56). Es precisamente en esta interacción cognitiva donde yace la posibilidad de que se dé la transacción estética que origina la respuesta a un texto dado.

En la transacción estética, el mensaje no verbal es igualmente significativo y se haya implícito, desde siempre, en el intercambio de imágenes y experiencias que supone el acto de comprensión lectora (Golden, J. 1985; Langer, J. 1989; Sadosky et al., 1990). Esto quiere decir que el papel del instructor -sea éste cualquier adulto involucrado en el proceso-, en el desarrollo de la comprensión lectora en el niño, es crucial. La acción de un buen maestro ayuda a acelerar la relación natural entre el lector aprendiz y el texto, relación que ha 
quedado demostrada en numerosos estudios con niños escolares y preescolares. Tanto Lindfors (1988) y Miller \& Gildea (1987), como Cochran-Smith (1986) concuerdan que esta disposición, materia prima que ha de ser procesada y pulida mediante la enseñanza formal de la comprensión de lectura, exige, de por sí, una reestructuración de las metodologías tradicionales.

\section{De la cognición a la transacción estética}

\author{
Sandy: Te has sentido alguna vez como el \\ protagonista? \\ Tamekia: Me sentí como su madre. Me \\ pregunté cómo crecería y estaría \\ preparada para el mundo real.
}

(En Spiegel, 1998, mi traducción).

La cita anterior describe una instancia típica de lo que ocurre en una clase de lectura en la que el enfoque metodológico trasciende lo meramente cognitivo. Se trata de la transacción estética con un texto literario por parte de dos lectoras de quinto grado. La teoría de la transacción estética de L.M. Rosenblatt, pionera del paradigma de la estética de la recepción, se resume en su libro The Reader, the Text, the Poem (1977). Su enfoque descansa en el supuesto de que el significado de un texto está en el lector quien, con la guía del texto y sus propias vivencias va creando el "poema". En su máxima expresión, esta interacción o transacción, como prefiere llamarla Rosenblatt, es una experiencia en la que lo racionalcognitivo se sujeta de lo transaccional-estético. El resultado de esta mutua dependencia queda manifiesta en la respuesta del lector, en su propia creación estética.

La teoría de la transacción, como marco metodológico complementario para la enseñanza de la lectura, impone un criterio de compromiso de orden superior por parte de los docentes para con los cientos de lectores que han de pasar por sus aulas. Como se ve, al privilegiarse la respuesta por sobre lo literal, la lectura y, específicamente, la lectura de textos literarios, trasciende lo meramente utilitarista. Ya el texto es algo más que una fuente de detalles puntuales que el lector debe identificar o recordar. Más bien, el acto de lectura se convierte en un evento vital; no en un medio para recopilar información solamente. En este sentido, Rosenblatt hace la diferencia entre la lectura de transacción "eferencial" (de effere, llevar afuera), y la lectura de transacción estética. La dirección que el lector asuma dependerá, entonces, de su posicionamiento en determinado momento durante el continumm del proceso. Así, la comprensión lectora puede involucrar los afectos, emociones y valores del lector como parte de la totalidad del evento, o bien, puede ser sólo un medio de trasmisión de información nueva con el texto como vehículo. Como esta doble dirección no puede darse simultáneamente, desde luego, la responsabilidad de crear un balance entre ambos modelos está en una instrucción más abierta a aquello de "mil lectores, mil lecturas". Frecuentemente, en las clases de lengua materna en nuestras escuelas, aún se tiende a poner más énfasis en lo eferencial, desestimándose el componente lúdico. Es posible que una de las numerosas causas de deserción escolar encuentre su raíz en esta carencia. Las cifras de deserción escolar, según consta en los informes emanados del Ministerio de Educación (ver página web), nos dicen que tres de cada diez niños abandonan la escuela primaria y esta cifra aumenta en la secundaria. Según ADA (Amigos del Aprendizaje en página web), la deserción va de la mano con la falta de comprensión lectora, lo que evidencia una falla en el enfoque tradicional. No es secreto que en la comprensión lectora reside el éxito académico en general. -Una pregunta necesaria en el contexto de esta discusión es si podría yacer en un enfoque meramente cognitivo o, en su defecto, meramente eferencial, uno de 
los múltiples factores que han incidido en que, por lo general, el costarricense no posea buenos hábitos de lectura, es decir, que no le interese leer por placer. -A modo de ilustración en el apéndice se ofrece una muestra de una tendencia metodológica tradicional reflejada en un examen típico de segundo grado.

En resumen, se pueden vislumbrar las implicaciones pedagógicas correspondientes a la lectura como experiencia estética, cuando comprendemos que ordinariamente ocurre lo contrario en el aula. En realidad, el enfoque de la lectura en aras de la respuesta del lector no es una práctica común en la aulas de escuelas y colegios, pese a los esfuerzos por modificar esta dirección unilateral. Este fenómeno no es nuevo, sino típico de nuestra tradición educativa. Para comenzar, la práctica de la lectura de textos tales como novelas y libros para jóvenes, es un fenómeno raro en nuestras escuelas primarias. Se tiende a sacrificar este disfrute en aras del cumplimiento de un programa a menudo saturado de contenidos. Dentro de estos parámetros metodológicos abundan las "guías" o cuestionarios en torno a algunos textos literarios, lo que equivale, dentro del contexto transaccional, a privilegiar el componente eferencial, que, como dijimos, excluye, de por sí, lo transaccional-estético. Este método es quizás el responsable de que las enseñanzas que puedan sacarse de un buen texto no se aprovechen para la vida. Pasado el "examen parcial", la mayor parte de la información se olvida por carecer de significado para el joven lector. En este sentido, cabe señalar la apreciación que otrora expresara R. Tyler (1973) sobre lo que él llamara "el olvido de lo aprendido". En su libro, Principios Básicos de Curriculo, Tyler hace mención de hallazgos realizados por un grupo de estudios entre estudiantes universitarios y señala que "la mitad del material conocido por el alumno al finalizar un curso se olvida al cabo de un año y la proporción olvidada a los dos años alcanza el 80\%” (p. 43). Los estudios a que hace mención el autor subrayan que una de las causas del olvido es la [carencia] de oportunidad de aplicar esos conocimientos a la vida cotidiana ..." (p. 43).

Las implicaciones pedagógicas de un giro comprometido en el enfoque de la enseñanza de la lectura como una experiencia lúdica, son evidentes. En primera instancia, el sólo hecho de enfocar la lectura dentro del paradigma de la respuesta del lector incentiva, de por sí, una transformación en la dirección metodológica. Los beneficios de la respuesta del lector como enfoque para la enseñanza de la lectura han quedado demostrados una y otra vez a través de la investigación empírica. Spiegel (1998) fundamenta esos beneficios en la naturaleza dinámica, reflexiva e introspectiva que supone la construcción del significado del texto durante el proceso estético-transaccional (p. 42), con el resultante desarrollo intelectual y personal del aprendiz. Un lector novato a quien se le somete a interactuar vitalmente con el texto, desarrollará destrezas analíticas que redundarán en un pensamiento más crítico y maduro. Lo anterior se verá reflejado tanto en lo que dice como en lo que escribe. Dugan (1997), Griffith \& Laframboise (1998) y Lancia (1997), concuerdan en que la lectura enfocada dentro de un marco transaccional tiene un efecto directo en las composiciones escritas de niños escolares desde etapas tempranas. La respuesta estética se refleja no sólo en la madurez de las ideas sino también en la estructuración de las mismas. Lancia, por ejemplo, encontró que ya desde segundo grado, los niños adquieren "préstamos" de patrones retóricos, vocabulario y estructuras sofisticadas que los pequeños escritores interiorizan y hacen suyas sin ayuda del maestro: "Nunca les he pedido a mis estudiantes [de segundo grado], escribe Lancia, escribir imitando un modelo literario. . . aunque algunos grupos han escrito historias siguiendo el patrón de libros que se leyeron 
en voz alta [en el aula]" (p. 470, mi traducción). Dugan, por su parte, le confiere al enfoque de la respuesta transaccional, en virtud de la naturaleza interpretativa de la misma, la cualidad de producir lectores más responsables: "Los niños expresan sus opiniones, preguntan por qué, y se desafían unos a otros para defender sus posiciones" (p. 94, mi traducción). Finalmente, las palabras de Spiegel resumen las virtudes de un enfoque transaccional de la lectura que a su vez hacen ver las profundas implicaciones pedagógicas del mismo. Dice:

"La investigación demuestra que los estudiantes crecen en al menos seis áreas en su habilidad para responder a la literatura. Ellos desarrollan autoría de lo que leen y de sus respuestas. Hacen conexiones personales con la literatura. Desarrollan una apreciación hacia las múltiples interpretaciones junto con una tolerancia y aún expectativas hacia la ambigüedad. Se hacen más reflexivos y críticos, trasladándose hacia niveles superiores del pensamiento y hacia un más rico entendimiento de la literatura" (pp. 4344, mi traducción).

\section{Conclusión}

Tragedia es una palabra fuerte, pero no puedo pensar en ninguna otra para describir lo que le pasa a la mayoría de los niños durante los primeros años de enseñanza primaria. En mis visitas a escuelas por todo el país, me he paseado una y otra vez del aula del kindergarden a la del cuarto grado... Y he hablado con cientos de educadores respecto a la aparentemente misteriosa discrepancia humana revelada a través de esa corta jornada. Es como si estuviéramos viendo especímenes de diferentes especies... Ve a una aula de kinderganden; generalmente, los niños de cinco años son espontáneos, únicos... Camina corredor abajo hacia los de cuarto grado; muy pronto notarás que algo se ha perdido ...

\section{(G. Leonard, Education and Ecstasy, 1987).}

La cita anterior resume de alguna manera, el pensamiento que respalda la discusión alrededor del tema de la comprensión lectora que se ha expuesto aquí. Uno puede argumentar, junto con Leonard, que este súbito cambio en la actitud de los niños escolares obedece al proceso natural de crecimiento porque, raramente, aparece un patrón diferente. Pero la pregunta sería: ¿deberá darse este proceso de esa manera necesariamente? Podemos también especular, como lo hace Leonard, acerca del intrigante fenómeno en relación con el hecho de que el potencial creativo e intelectual del niño parece decaer en cuanto éste entra a la escuela, hecho que Leonard no considera casual.

Es bien sabido que el medio cultural y social que rodea al niño es el generador primordial del desarrollo del lenguaje en todas sus formas, pero también existe una necesidad casi instintiva en el niño de "husmear" en el material impreso, tendencia esta que responde a un mecanismo en su cerebro que trasciende lo meramente cognitivo. En realidad, en las letras y los dibujos los niños ven su propio mundo interior impreso. De aquí, su habilidad para captar el lenguaje metafórico y la fábula. Sin embargo, la enseñanza programada a veces parece ignorar estas importantísimas relaciones de orden cognitivo y estético, provocándose un desajuste entre los métodos didácticos y la lógica del niño en lo que cabe a su proceso de comprensión lectora. Es decir, así como la enseñanza programada de la lectura es esencial en la totalidad del proceso de aprendizaje, también puede ser dañina si no parte de los supuestos adecuados. Desafortunadamente, la instrucción formal tradicional abunda, en muchos casos, en técnicas "cuasi-conductistas" que obligan al niño a distanciarse de la lengua en etapas en las que éste está aún lejos de haber desarrollado una conciencia meta-lingüística. Casi podría decirse que muchos niños aprenden a leer a pesar de y no por los métodos tradicionales. Lo anterior debe obligarnos a meditar en lo que hubiera sido de habérseles dado la oportunidad de desarrollar simultáneamente sus potenciales intelectual y creativo, mediante técnicas alternativas más cercanas a la naturaleza lúdica del proceso 
natural de adquisición del lenguaje. El enfoque apoyado tan sólo por el método cognitivo se apoya, por definición, en el hecho fundamental de que la adquisición del lenguaje es innata, pero parece contrariar ese hecho cuando en la práctica, se subestima el principio de que la lectura y la escritura son sólo una reproducción arbitraria de aquella condición innata. Y es que el cerebro joven parece estar listo para dar sólo un paso a la vez en el proceso de decodificación de su mundo natural mediante símbolos. La verdad es que este proceso, ya de por sí violento, le significa al niño una relación meta-lingüística con su entorno para la que hay que prepararlo gradualmente. Se infiere entonces que, si además de esta "distancia obligada", se enfrenta a los niños con técnicas de lectura inadecuadas, las consecuencias graves en el proceso de enseñanza-aprendizaje, en general, son perfectamente predecibles. Generación tras generación de niños escolares y adolescentes arrastran las secuelas de una mala instrucción en la enseñanza de la lengua materna, verdad que se pone en evidencia cuando se enfrenta más tarde al cerebro desentrenado con textos que requieren de una lectura crítica.

La comprensión lectora sigue siendo una interrogación abierta en términos de la dirección didáctica que a menudo se le da. A menudo lo maestros parecemos ignorar que el lenguaje está en constante evolución en el sentido chomskiano. La lectura de un texto literario, por ejemplo, debe presuponer también una experiencia estética y vital distinta cada vez. Tal es la naturaleza del lector. Pero como cualquier otra actividad del conocimiento humano, la lectura estética requiere entrenamiento. Es decir, se aprende a leer y a disfrutar de la lectura, leyendo. Para esto, una revisión de las normas y políticas metodológicas existentes en torno a la enseñanza de la lectura no es sólo necesaria, sino insoslayable y requiere un verdadero compromiso por parte de todos los involucrados en el rumbo que la educación, en materia de enseñanza de la lengua materna, debe tomar.

\section{Referencias bibliográficas}

Adams, Marilyn. Beginning to Read. Cambridge: The MIT Press. 1990.

Amigos del Aprendizaje, www.ADA.or.cr.

Applebee, Arthur. The Child's Sense of Story. Chicago: The University of Chicago Press. 1989.

Chomsky, Noam. "How language is shaped”. En P. Richard-Amato, Making it Happen: Interaction in the Second Language Classroom, Theory and Practice. New York: Longman. 1988.

Cochran-Smith, Patricia. "Reading to children: A model for understanding texts". En B. Schiefflin \& P. Elmore (Eds). The acquisition of Literacy: Ethnographic Perspectives. New York: Abblax, 1986.

Downing, John. Reading and Reasoning. New Jersey: Springer-Velarg. 1979.

Golden, Jane. "Interpreting a tale". Poetics. Vol. 14, pp. 503-24. EUA.1985.

Leonard, George. Education and Ecstasy. New York: Longman. 1987.

Lindfors, Jane. Children's Language and Learning. New York: Prentice Hall. 1988.

Ministerio de Educación Pública. (www.mep. go.cr/cuadrodesercióngeneral)

Miller, G. \& Gildea, P.M. "How children learn words." Scientific American, Vol. 257, No. 3, pp. 94-99. EUA. 1987. 
Pearson, David. "The comprehension of metaphor by preschool children". Journal of Child Language. Vol. 17, No. 1, pp. 185-201. EUA. 1996.

Rosenblatt, Louise Marie. The Reader, The Text, The Poem. Carbondale: Southern Illinois. University Press. 1977.

Rumelhart, David. "Schemata: the building blocks of cognition". In Spiro, R.J. et al. (Eds), Theoretical Issues in Reading Comprehension. New Jersey: Lawrence, Erlbaum, Associates. New Jersey. 1977.

Sadosky, Mark, Goetz, E. \& Olivares, M. "Imagination in story reading: the role of imagery, verbal recall, story analysis and processing levels." Journal of Reading Behavior. Vol. 22, No. 1, 55-70. EUA. 1990.

Sánchez, Marta. "Reading literary works in a foreign language: transaction and Interaction". Revista de Filología y Lingüística, XIX (2), 63-68. Costa Rica. 1993.

Sánchez, Marta. "La teoría de los esquemas y la comprensión de lectura: algunas consideraciones generales". Káñina, XIX (1), 137-142. Costa Rica. 1995.
Sánchez, Marta. "El proceso de comprensión lectora: un estudio comparativo entre lectores de lengua extranjera y lectores de lengua materna”. Káñina, XXII (1), 95-106. Costa Rica. 1998.

Sánchez, Marta. "Los efectos de los esquemas lingüístico-culturales en la transacción texto-lector durante el proceso de lectura de un texto literario en una lengua extranjera". Repertorio Americano 8, 81-95. Costa Rica. 1999.

Sánchez, Marta. "El uso de textos literarios en la enseñanza de la composición en una lengua extranjera: una propuesta metodológica respaldada por la teoría del esquema y la teoría de la transacción estética de L.M. Rosenblatt”. Repertorio Americano. Costa Rica. 2000.

Smith, Frank. Understanding Reading. New Jersey: Lawrence Erlbaum, Associate Publishers. 1988.

Strange, Mark. "Instructional implications of a conceptual theory of reading comprehension". Reading Teacher. Vol. 33, pp. 391-397. EUA. 1980.

Tyler, Robert. Principios Básicos del Currículo. Chicago. University of Chicago, Press. Chicago. EUA. 1973. 


\section{Apéndice}

Muestra parcial de un examen de español de segundo grado con texto literario. [Primera prueba de español, Escuela María Auxiliadora]

\section{INSTRUCCIONES GENERALES:}

Lea con atención lo que se le solicita. No tache, no manche sus respuestas. Conteste únicamente lo que se le solicita.

\section{LEA CON MUCHA ATENCIÓN:}

\section{¡Llueve!}

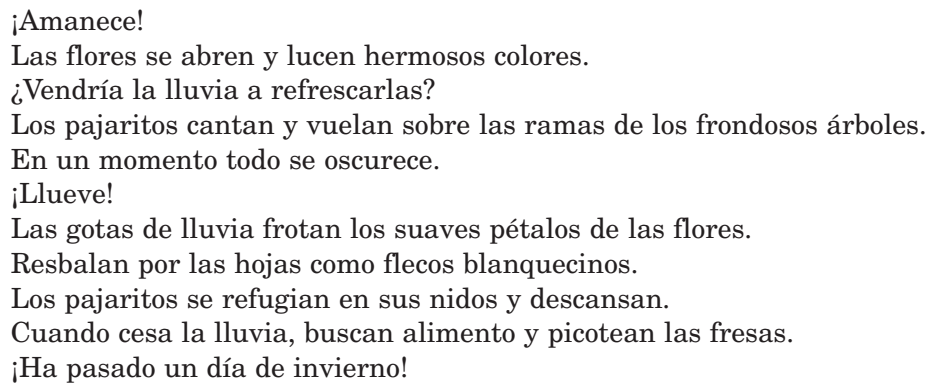

I PARTE. INTERROGATIVA [. . .] CONTESTE LAS SIGUIENTES PREGUNTAS CON ORDEN Y CLARIDAD SEGÚN SE LE PREGUTA AYUDADA DEL TEXTO ANTERIOR.

¿Qué le [sic] sucede a las flores cuando amanece?

¿Qué hacen los pajaritos?

¿Qué frotan las gotas de lluvia?

¿Por dónde resbalan las gotas de lluvia?

[ETC]

\section{IDENTIFICACIÓN.}

INSTRUCCIONES:

A. Divido las siguientes palabras [en] sílabas. Coloreo . . . la sílaba tónica.

\section{COCHE CARRETA DIRIGIBLE [ETC]}

B. Identifico en la siguiente lista de palabras ubicadas en los sombreros, los hiatos, coloreando el sombrero de color azul, y los diptongos coloreando el sombrero de color verde.

$$
\text { llueve vecindario piruetas [ETC....] }
$$


III PARTE. COMPLETE

INSTRUCCIONES

Se le presentan unas palabras con un espacio en blanco y una nubecitas con la sílaba que usted debe colocar en ese espacio según corresponda.
1. ma
2. re so
3.
llo
4. Mila

GRA GRE GRI GRO [ETC....]

Marta E. Sánchez Salazar

Coordinadora de la Sección de Inglés para otras Carreras de la Escuela de Lenguas Modernas de la Universidad de Costa Rica 
